\title{
INDECOMPOSABLE CONTINUA IN STONE-ČECH COMPACTIFICATIONS
}

\author{
DAVID P. BELLAMY AND LEONARD R. RUBIN
}

\begin{abstract}
We show that if $Y$ is a continuum irreducible from $a$ to $b$, which is connected im Kleinen and first countable at $b$, and if $X=Y-\{b\}$, then $\beta X-X$ is an indecomposable continuum. Examples are given showing that both first countability and connectedness im Kleinen are needed here. We also show that $\beta[0,1)-[0,1)$ has a strong near-homogeneity property.
\end{abstract}

1. Introduction. In [2] and [3] it is shown that if $X=[0,1)$ then $\beta X-X$ is an indecomposable continuum; here $\beta X$ is the Stone-Cech compactification of $X$. In [7], Dickman showed that among locally connected spaces, $[0,1)$ is essentially the only such space. In this paper we exhibit other types of spaces $X$ with this property. We shall also show that for $X=[0,1)$, $\beta X-X$ is stably almost homogeneous, a concept to be defined below.

The set function $T$ has been studied and applied in [1], [5], [6], [8], [9], [11], and [14]. We follow these papers in writing $T(p)$ for $T(\{p\})$. This set function will be used in the argument at one point and familiarity with it is assumed. Familiarity with [10], [12], [13], and [15] is also assumed. If we write $X=A \cup B$ sep, then we mean that $\mathrm{Cl}(A) \cap B=\varnothing$ and $A \cap \mathrm{Cl}(B)=\varnothing$ while neither $A$ nor $B$ is empty. By $f: X \cong Y$, we mean $f$ is a homeomorphism of $X$ onto $Y$.

\section{Indecomposable continua in $\beta X$.}

LEMMA 1. There is a covariant functor $\beta$ on the category of Tychonoff spaces and continuous maps such that for any Tychonoff space $X, \beta X$ is the Stone-Cech compactification of $X$ and if $f: X \rightarrow Y$ then $\beta f: \beta X \rightarrow \beta Y$ is the unique extension of $f$ induced by $f$ treated as a map from $X$ to $\beta Y$.

Notation. If $X$ is a Tychonoff space, let $\gamma X=\beta X-X$. If $f$ is a continuous map from $X$ to $Y$, let $\gamma f$ denote $\beta f \mid \gamma X$.

DEFINITION. Let $Y$ be a compact Hausdorff continuum irreducible from $a$ to $b$ such that $Y$ is both connected im Kleinen and first countable

Received by the editors November, 1971 and, in revised form, September 8, 1972.

AMS (MOS) subject classifications (1970). Primary 54C05, 54D35, 54F20; Secondary 54F15.

Key words and phrases. Remainders of compactifications.

(c) American Mathematical Society 1973 
at $b$. Let $X=Y-\{b\}$. Then we call the topological pair $(Y, X)$ a wave from $a$ to $b$.

By stringing together indecomposable continua, a wave $(Y, X)$ can be constructed such that $Y$ is not connected im Kleinen at any point of $X$.

LEMMA 2. If $Y$ is a compact Hausdorff continuum irreducible from a to $b$ and $x \in Y, T(x)$ either separates a from $b$, contains $a$, or contains $b$. In case $T(x)$ separates a from $b, Y-T(x)$ has exactly two components, $A$ and $B$, where $a \in A$ and $b \in B$, and both $T(x) \cup A$ and $T(x) \cup B$ are proper subcontinua of $Y$ containing $a$ and $b$ respectively.

REMARK ON PROOF. This lemma can be established using standard techniques and Theorem 1.10 of [14], since each $x \in Y$ different from $a$ and $b$ weakly separates $a$ from $b$.

LEMMA 3. If $Y$ is a compact Hausdorff continuum irreducible from $a$ to $b$ and $W \subseteq Y$ is a continuum with $b \in$ Int $W$, then $W-\{b\}$ is connected.

Proof. If $W-\{b\}=M_{0} \cup N_{0}$ sep, let $M=M_{0} \cup\{b\} ; N=N_{0} \cup\{b\}$. Then $b$ lies in the boundary of $M$ and $N$ and, by Theorem 6 of [15, p. 194], each of $M$ and $N$ is nowhere dense, so that $M \cup N=W$ is nowhere dense also, a contradiction.

LEMMA 4. If $(Y, X)$ is a wave from a to $b$, and $Z$ is a Hausdorff compactification of $X$, then $Z-X$ is a Hausdorff continuum.

Proof. Since $Y$ is connected im Kleinen and first countable at $b$, there exists a denumerable collection of continua $\left\{N_{i}\right\}_{i=1}^{\infty}$ such that for each $i, b \in \operatorname{Int}\left(N_{i}\right)$ and $N_{i+1} \subseteq N_{i}$ and $\bigcap_{i=1}^{\infty} N_{i}=\{b\}$. It is readily seen that

$$
Z-X=\mathrm{Cl}_{Z}\left(N_{i}-\{b\}\right)-N_{i}=\bigcap_{i=1}^{\infty} \mathrm{Cl}_{Z}\left(N_{i}-\{b\}\right) .
$$

Then, since each $N_{i}-\{b\}$ is connected by Lemma $3, Z-X$ is an intersection of a monotone collection of continua.

Lemma 5. Let $X$ be a compact Hausdorff space, $b \in X,\{b\}$ a component of $X$, and suppose $X$ is first countable at $b$ and $\left\langle b_{i}\right\rangle_{i=1}^{\infty}$ is a sequence in $X-\{b\}$ converging to $b$. Then there exist two closed subsets $A$ and $B$ of $X$ such that $A \cup B=X, A \cap B=\{b\}$, and each of $A$ and $B$ contains infinitely many (that is, a subsequence) of the $b_{i}$ 's.

Proof. It is readily seen that there exists a neighborhood basis $\left\{N_{j}\right\}_{j=1}^{\infty}$ at $b$ consisting of closed and open sets such that $N_{j+1} \subseteq N_{j}$ for each $j$ and $N_{1}=X$; by passing to a subset if necessary, we may suppose that each 
$N_{j}-N_{j+1}$ contains at least one of the $\mathrm{b}_{i}$ 's. Then set

$$
A=\{b\} \cup \bigcup_{j=1}^{\infty}\left(N_{2 j-1}-N_{2 j}\right), \quad B=\{b\} \cup \bigcup_{j=1}^{\infty}\left(N_{2 j}-N_{2 j+1}\right) .
$$

Then $A$ and $B$ have the desired properties.

LEMMA 6. If $(Y, X)$ is a wave from $a$ to $b$ and $W$ is a nondegenerate subcontinuum of $Y$ containing $b$, then $b \in$ Int $W$.

Proof. Suppose not. Then let $p \in W, p \neq b$. Since $b \notin T(p)$, by connectedness im Kleinen at $b$, it follows that either $a \in T(p)$ or $Y-T(p)=$ $A \cup B$ sep, where $a \in A$ and $b \in B$. If $a \in T(p), T(p) \cup W$ is a proper subcontinuum of $Y$ containing both $a$ and $b$; if $a \notin T(p), A \cup T(p) \cup W$ is such a continuum, and in either case we have a contradiction.

COROllary 1. If $(Y, X)$ is a wave from a to $b$ and $M$ is a closed subset of $Y$ with $b \in M$ but $b \notin$ Int $M,\{b\}$ is a component of $M$.

LEMMA 7. If $Y$ is a compact Hausdorff space first countable at a point b, then $Y-\{b\}$ is normal.

Proof. Let $\left\{O_{k}\right\}_{k=1}^{\infty}$ be a countable basis of open neighborhoods at $b$. Then $Y-\{b\}=\bigcup_{k=1}^{\infty}\left(Y-O_{k}\right)$, so that $Y-\{b\}$ is sigma compact and hence Lindelöf. Then $Y-\{b\}$ is paracompact $[10$, p. 174, 6.5] and hence normal $[10$, p. $163,2.2]$.

THEOREM 1. If $(Y, X)$ is a wave from a to $b$, then $\gamma X$ is an indecomposable continuum.

Proof. By Lemma $4, \gamma X$ is a continuum. Suppose $F$ is a proper subcontinuum of $\gamma X$ which contains an interior point $q$ with respect to $\gamma X$. Let $p \in \gamma(X)-F$. Let $U$ and $V$ be open sets in $\beta X$ with $\mathrm{Cl}(U) \cap \mathrm{Cl}(V)=$ $\mathrm{Cl}(U) \cap(\gamma X-\operatorname{Int} F)=\mathrm{Cl}(V) \cap F=\varnothing$ while $p \in V$ and $q \in U$. This is possible by regularity.

Then $X \cap V$ and $X \cap U$ are open subsets of $X$ and hence of $Y$ since $X$ is open in $Y$. Let $\left\langle b_{i}\right\rangle_{i=1}^{\infty}$ be a sequence of points in $U \cap X$ converging in $Y$ to $b$. This is possible since $b \in \mathrm{Cl}_{Y}(U \cap X)$ and $Y$ is first countable at $b$.

Then $\{b\}$ is a component of $Y-(V \cap X)$, by Corollary 1 , and $\left\langle b_{i}\right\rangle$ is a sequence in $(Y-V)-\{b\}$ converging to $b$. By Lemma 6 there are two closed sets $A_{0}$ and $B_{0}$ such that $A_{0} \cup B_{0}=Y-V, A_{0} \cap B_{0}=\{b\}$, and each of $A_{0}$ and $B_{0}$ contains a subsequence of the $b_{i}$ 's. Let $A=A_{0} \cap X, B=B_{0} \cap X$. Then $A$ and $B$ are disjoint closed subsets of $X$, and since $X$ is normal, disjoint closed sets lie in disjoint zero sets, and by Theorem 6.5 III of [12], $\mathrm{Cl}_{\beta X}(A) \cap \mathrm{Cl}_{\beta X}(B)=\varnothing$. Now since each of $A$ and $B$ contains infinitely many of the $b_{i}$ 's, it follows that each of $\mathrm{Cl}_{\beta X}(A)$ and $\mathrm{Cl}_{\beta X}(B)$ contains 
points of $\mathrm{Cl}_{\beta X}(U) \cap \gamma X$, and hence points of $F$. Thus, since if $x \in \gamma X-$ $\mathrm{Cl}_{\beta X}(A \cup B)$, it follows that $x \in \mathrm{Cl}_{\beta X}(V)$ and hence $x \notin F$, we have $F=$ $\left(F \cap \mathrm{Cl}_{\beta X}(A)\right) \cup\left(F \cap \mathrm{Cl}_{\beta X}(B)\right)$ sep, so that $F$ is no continuum.

Corollary 2 ([2] AND [3]). Let $X=[0,1)$. Then $\gamma X$ is an indecomposable continuum.

EXAMPLE 1. Let $L$ denote the long line, consisting of $\omega_{1} \times[0,1)$ with the lexicographic order, where $\omega_{1}$ is the first uncountable ordinal; we take the order topology on $L$. Then consider $L \times[0,1]$ with the product topology. Let

$$
X=\{((\alpha, t), s) \in L \times[0,1]: t=0 \text { or } t=s\} .
$$

Let $Y=X \cup\{b\}$ be the one-point compactification of $X$. Then $Y$ is irreducible from $((0,0), 1)$ to $b$ and is connected im Kleinen at $b .(Y, X)$ fails to be a wave from $a$ to $b$ because $Y$ is not first countable at $b$.

Standard techniques applied to continuous functions from $\omega_{1}$ to $[0,1]$ yield the result that $\gamma X \cong[0,1]$ in this case. Thus, first countability cannot be dispensed with in the hypothesis of Theorem 1. Connectedness im Kleinen also cannot be removed from the hypothesis of Theorem 1; the usual topologist's $\sin 1 / x$ curve, with $b$ taken from the limit arc, yields a decomposable continuum as $\gamma X$.

LEMMA 8. If $X$ is a Tychonoff space and $Z$ is any compactification of $X$ with inclusion map $i: X \rightarrow Z$, then $\gamma i(\gamma X)=Z-i(X)$.

REMARK ON PROOF. This is a special case of Theorem 6.12 of [12, p. 92].

Lemma 9. If $X$ and $Y$ are Tychonoff spaces and $f: X \cong Y$, then $\gamma f$ : $\gamma X \cong \gamma Y$.

Proof. By Lemma $8, \gamma f(\gamma X)=\gamma Y$ and since $\beta$ is a functor it follows that $\beta f$ is a homeomorphism since it has inverse $\beta\left(f^{-1}\right)$. Then $\gamma f$ is a homeomorphism since it is a restriction of one.

Lemma 10. Let $X$ be a normal Hausdorff space and A a closed subset of $X$ such that $X-A$ contains $a$ closed but not compact subset of $X$. Then $\gamma X-\mathrm{Cl}_{\beta X}(A)$ is a nonempty, open subset of $\gamma X$.

Lemma 11. Suppose $X$ is a Tychonoff space and $f: X \cong X$ is the identity inside some closed subset $V$ of $X$. Then $\gamma f: \gamma X \cong \gamma X$ is the identity inside $\gamma X \cap \mathrm{Cl}_{\beta X}(V)$.

Definition. We say a topological space $X$ is almost homogeneous if for any $p, q \in X$, and any neighborhood $U$ of $q$ there is a homeomorphism $h: X \cong X$ such that $h(p) \in U$. If, in addition, we may choose $h$ to be the 
identity on some nonempty open subset of $X$, we say $X$ is stably almost homogeneous.

THEOREM 2. Let $X=[0,1)$; then $\gamma X$ is a stably almost homogeneous indecomposable continuum.

Proof. Throughout this proof, $\mathrm{Cl}$ denotes $\mathrm{Cl}_{\beta X}$. Let $x, y \in \gamma X$ and let $V_{0}$ be any open set in $\gamma X$ containing $y$. Then $V_{0}=V_{1} \cap \gamma X$ for some $V_{1}$ open in $\beta X$. Then there exists a $V_{2}$ open in $\beta X$ such that $y \in V_{2} \subseteq \mathrm{Cl} V_{2} \subseteq V_{1}$ and $x \notin \mathrm{Cl} V_{2}$ unless $x=y$, in which case there is nothing to prove. Let $U_{0}$ be open in $\beta X$ with $x \in U_{0}$ and $\mathrm{Cl} U_{0} \cap \mathrm{Cl} V_{2}=\varnothing$. Now let $U=U_{0} \cap X$ and $V=V_{2} \cap X$. We shall assume, with no loss of generality, that $0<\inf U<$ $\inf V$.

Now, define four sequences $\left\langle p_{n}\right\rangle_{n=1}^{\infty},\left\langle q_{n}\right\rangle_{n=1}^{\infty},\left\langle r_{n}\right\rangle_{n=1}^{\infty}$, and $\left\langle s_{n}\right\rangle_{n=1}^{\infty}$ as follows: $p_{1}=\inf U$. Whenever $p_{i}$ has been defined, set $q_{i}=\sup \{t \in U$ : $\left.\left[p_{i}, t\right] \cap V=\varnothing\right\}$. When $q_{i}$ has been defined, set $r_{i}=\inf \left\{t \in V: t>q_{i}\right\}$. When $r_{i}$ has been defined, set $s_{i}=\sup \left\{t \in V:\left[r_{i}, t\right] \cap U=\varnothing\right\}$. When $s_{i}$ has been defined, set $p_{i+1}=\inf \left\{t \in U: t>s_{i}\right\}$. This completes the recursive definition of the four sequences. They have the following properties: $p_{i}<q_{i}<r_{i}<s_{i}<$ $p_{i+1}$ for each $i$; the limit in $[0,1]$ of each sequence is $1, U \subseteq \bigcup_{i=1}^{\infty}\left[p_{i}, q_{i}\right]$, and $V \subseteq \bigcup_{i=1}^{\infty}\left[r_{i}, s_{i}\right]$. We now choose two more sequences $\left\langle x_{i}\right\rangle_{i=1}^{\infty}$ and $\left\langle y_{i}\right\rangle_{i=1}^{\infty}$ so that, for each $i, r_{i}<x_{i}<y_{i}<s_{i}$ and the closed interval $\left[x_{i}, y_{i}\right]$ is a subset of $V$. Finally we choose two more sequences $\left\langle a_{i}\right\rangle_{i=1}^{\infty}$ and $\left\langle b_{i}\right\rangle_{i=1}^{\infty}$ such that $a_{1}=0 ; 0<b_{1}<p_{1}$, and for $i>1$ we choose $s_{i}<a_{i+1}<b_{i+1}<p_{i+1}$. Now define $h: X \cong X$ as follows: For each $i$,

(1) $h$ is the identity on $\left[a_{i}, b_{i}\right]$,

(2) $h$ maps the interval $\left[b_{i}, p_{i}\right]$ linearly onto $\left[b_{i}, x_{i}\right]$,

(3) $h$ maps $\left[p_{i}, q_{i}\right]$ linearly onto $\left[x_{i}, y_{i}\right]$,

(4) $h$ maps $\left[q_{i}, a_{i+1}\right]$ linearly onto $\left[y_{i}, a_{i+1}\right]$.

Then $h(U) \subseteq V$, and hence $\beta h(\mathrm{Cl}(U)) \subseteq \mathrm{Cl}(V)$, and since $x \in \mathrm{Cl}(U)$, $\beta h(x) \in \mathrm{Cl}(V) \subseteq \mathrm{Cl}\left(V_{2}\right) \subseteq V_{1}$, and $\beta h(x)=\gamma h(x) \in V_{0}$ as desired. Furthermore, $\gamma h$ is the identity inside the set $\gamma X \cap \mathrm{Cl}\left(\cup_{i=1}^{\infty}\left[a_{i}, b_{i}\right]\right)$, which contains a nonvoid open subset of $\gamma X$ by Lemma 10, setting the closed set $\bigcup_{i=1}^{\infty}\left[b_{i}, a_{i+1}\right]$ equal to $A$ in the lemma.

\section{REFERENCES}

1. D. P. Bellamy, Continua for which the set function $T$ is continuous, Trans. Amer. Math. Soc. 151 (1970), 581-587. MR 42 \#6791.

2. - A non-metric indecomposable continuum, Duke Math. J. 38 (1971), 15-20. MR 42 \#6792.

3. - Topological properties of compactifications of a half-open interval, $\mathrm{Ph} . \mathrm{D}$. Thesis, Michigan State University, East Lansing, Mich., 1968.

4. H. S. Davis, A note on connectedness im Kleinen, Proc. Amer. Math. Soc. 19 (1968), 1237-1241. MR 40 \#8021. 
5. H. S. Davis, D. P. Stadtlander and P. M. Swingle, Properties of the set functions $T^{n}$, Portugal. Math. 21 (1962), 113-133. MR 25 \#5501.

6. - Semigroups, continua, and the set functions $T^{n}$, Duke Math. J. 29 (1962), 265-280. MR 26 \#4325.

7. R. F. Dickman, Jr., A necessary and sufficient condition for $\beta X-X$ to be an indecomposable continuum, Proc. Amer. Math. Soc. 33 (1972), 191-194.

8. R. F. Dickman, Jr., L. R. Rubin and P. M. Swingle, Characterization of n-spheres by an excluded middle membrane principle, Michigan Math. J. 11 (1964), 53-59. MR 28 \#4523.

9. - Irreducible continua and generalization of hereditarily unicoherent continua by means of membranes, J. Austral. Math. Soc. 5 (1965), 416-426. MR 32 \#6424.

10. J. Dugundji, Topology, Allyn and Bacon, Boston, Mass., 1966. MR 33 \#1824.

11. R. W. Fitzgerald and P. M. Swingle, Core decompositions of continua, Fund. Math. 61 (1967), 33-50. MR 36 \#7110.

12. L. Gillman and M. Jerison, Rings of continuous functions, Van Nostrand, Princeton, N.J., 1960. MR 22 \#6994.

13. J. G. Hocking and G. S. Young, Topology, Addison-Wesley, Reading, Mass., 1961. MR 23 \#A2857.

14. R. P. Hunter, On the semigroup structure of continua, Trans. Amer. Math. Soc. 93 (1959), 356-368. MR 22 \#82.

15. K. Kuratowski, Topologie. Vol. 2, 3rd ed., Monografie Mat., Tom 21, PWN, Warsaw, 1961; English transl., Academic Press, New York; PWN, Warsaw, 1968. MR 24 \#A2958; MR 41 \#4467.

Department of Mathematics, University of Delaware, Newark, Delaware 19711

Department of Mathematics, University of Oklahoma, Norman, Oklahoma 73069 